\title{
SOFTWARE AGENTS AS LEGAL PERSONS
}

\author{
Francisco Andrade ${ }^{1}$, José Neves ${ }^{2}$, Paulo Novais ${ }^{2}$ and José Machado ${ }^{2}$ \\ ${ }^{1}$ Escola de Direito, Universidade do Minho, Braga, PORTUGAL \\ ${ }^{2}$ Departamento de Informática, Universidade do Minho, Braga, PORTUGAL \\ \{fandrade, jneves,pjon,jmac $\} @$ di.uminho.pt
}

\begin{abstract}
The Law has long been recognizing that, besides natural persons, other entities socially engaged must also be subject of rights and obligations. Western laws usually recognize Corporate Bodies as having legal personality and capacity for every right and obligation needed or convenient to the prosecution of its social goals. But can we foresee a similar attribution of such a regime to software agents? In other words, are intelligent software agents capable of being personified? One of the main characteristics of a personality is the existence of a physical being or organization provided with its own will. In that sense, intelligent software agents are quite close to human beings. Indeed, they have a physical existence, and they have the capability of learning and of having a will of their own.
\end{abstract}

\section{INTRODUCTION}

In order to evaluate the chances of attributing a legal personality to intelligent software agents, it might be interesting to analyse - establishing some due comparisons - the arguments that justified the consideration of corporate bodies as legal persons (Gonçalves, 1929).

In fact, legal persons are to be seen as a Technical Reality (Fernandes, 1995) or instrument at the service of The Law, through which it is achieved a way of dealing with certain human interests (Fernandes, 1995). Legal persons are thus considered a reality of the legal world corresponding to a social need, to a social interest worth of being dealt with, according to The Law. Applying such considerations to intelligent software agents, it may be argued that those are physical and logical entities capable of multiple and autonomous intervention in the legal world, whose personification under The Law might be foreseen as a technical way of responding to a social need - the need for more efficient and reliable ways of undertaking actions that man alone can not perform, or can not perform sufficiently and economically and in time.

Besides one own will, two basic requirements were enounced as needed for a corporate body to become a personality, and those were substratum (e.g., personal or 
patrimonial component, teleological component, intentional component) and recognition (Andrade, 1974). Does substratum exist in software agents? Can we consider its physical and logical structures as a personal or patrimonial element? Can we speak of teleological and intentional elements when referring to software? And how could recognition of legal personality to software substratum be handled?

The attribution of legal personality to intelligent software agents would have some obvious advantages: it would solve the question of consent and the validity of declarations and contracts enacted or concluded by software agents (Felliu) (Fisher, 2001); and it would reassure the owners-users of the agents about eventual liability concerns (Sartor, 2002). But it would also face several difficulties, due to the intrinsic characteristics of software agents - some difficult problems could arise relating to questions such as domicile or patrimony (Weitzenboeck) (Lerouge). And of course we must wonder whether electronic agents could or not be liable for negligent acts or omissions, whether it is possible or not to consider them to act in good or bad faith (Miglio et al), whether or not it is possible to sue a software agent in Court, or to impose sanctions on it (Andrade and Neves, 2004).

The attribution of legal personality to electronic agents would require at least some sort of constitution/declaration act and eventually registration (Allen et al, 1996), in order to attribute a physical location to the agent, a minimum patrimony through a banking deposit or even a compulsory insurance regime, in order to fulfill financial obligations and liabilities. But even if all those difficulties could be overcome, would it be worth such a legal attribution? Or should we rather foresee the creation of special corporate bodies on whose behalf the electronic agents would act? Anyway, we must have a realistic approach to this issue, considering the challenging technical possibilities of software agents as entities requiring a particular new legal setting in order to enhance the full use of e-Commerce in a global world.

Much work has been done in terms of the humanization of the behaviour of virtual entities, by expressing human like feelings and emotions; work presented in (Ortony et al, 1988) (Picard et al., 1997) detail studies and propose lines of action that consider the way to assign emotions to machines. Attitudes like cooperation, competition and socialization of agents (Bazzen at all, 2000) are explored, for example, in the areas of Economy (Arthur, 1994) and Physics (Challet and Zhang, 1998), as it is the case of the El Farol Bar Problem, the Minority Game and the Iterated Prisoner's Dilemma. In (Bazzen and Bordini, 2000) and (Castelfranchi et al., 1997) it is recognized the importance of modelling the virtual agent's mental states in a human like form.

Indeed, an important motivation to the development of this work comes from the one that is going on in the intersection of the disciplines of AI and The Law, that enforced new forms of knowledge representation and reasoning in terms of an extension to the language of logic programming (i.e., the Extended Logic Programming (ELP) (Alferes at all, 1998) (Neves, 1984) (Traylor and Gelfond, 1993) (Costa at al., 2000) (Costa and Neves, 2000). This introduction raised a very wide scientific area and raises a substantial number of issues. However, once one has limited space, the links between the key discussions that are addressed in the paper, will be set in terms of an analyse of the predicate reputation(). The formalization presented in chapters 3 and 4, which is aimed at reputation, intends to 
open the way to deal with legal issues, in a similar form. Indeed, reputation, in itself, may also be understood as a legal issue.

\section{MULTIAGENT SYSTEMS}

In the current economical context, characterized by the existence of a global society, the access to information is crucial for any economical and social development, still remain important technological challenges. The representation, maintenance and querying of information is a central part of this problem. How can we obtain the adequate information at the adequate time? How can we supply the correct items for the correct people at the correct time? How and where can we get the relevant information for a good decision making? The organizations focus their competences in strategically areas and have recourse to external supplies, cooperating with sporadically partners, with the objective of reducing costs, risks and technological faults or maximizing benefits and business opportunities. One of the most radical and spectacular changes is the information unmaterialization, the task or procedure automation, the recourse to decision support systems or intelligent systems and to new forms of celebrating contracts (e.g., is it possible to practice commercial acts and celebrate deals using autonomous and pro-active computational agents?). The eCommerce has now new challenges, searching for new answers to old questions. The negotiation processes through electronic means and the e-Commerce platforms may set new forms of contracts, with engagements and negotiations among virtual entities.

Software agents are computational entities with a rich knowledge component, having sophisticated properties such as planning ability, reactivity, learning, cooperation, communication and the possibility of argumentation. The use of the agent figure is particularly adequate to such problems. The objective is to build logical and computational models, as well as implementing them, having in consideration The Law norms (i.e., legislation, doctrine and jurisprudence). Agent societies may mirror a great variety of human societies, such as commercial societies with emphasis to behavioural patterns, or even more complex ones, with pre-defined roles of engagement, obligations, contractual and specific communication rules.

The traditional programming languages do not support the description of certain types of behaviour which usually involves computational agents. In genesis, systems that incorporate those functionalities have a multi-layer architecture, evolve from esoteric software sub-systems, network protocols, and the like. On the other hand, once one deals with multi-agent systems, it must be guaranteed that they may answer to different and simultaneous demands, in a secure and error free way. An agent must be able to manage its knowledge, beliefs, desires, intentions, goals and values. It may be able also to plan, receive information or instructions, or react to environment stimulus. It may communicate with others agents, share knowledge and beliefs, and respond to other agents upon request. It may cooperate diagnosing errors or information faults in its knowledge bases, sharing resources, avoiding undesirable interferences or joining efforts in order to revisit the knowledge bases of its own and of its peers, in order to reach common goals. 
Knowledge and belief are generally incomplete, contradictory or error sensitive, being desirable to use formal tools to deal with the problems that arise from the use of incomplete, contradictory, imperfect, wrong, nebulous or missing information. The Extended Logic Programming language presents itself as a formal and flexible tool to contribute to obtain a solution for the problems just referred to above.

\section{AN AGENT KNOWLEDGE BASE}

Using knowledge representation techniques as a way to describe the real world, based on mechanical, logical or other means will be, always, a function of the systems ability to describe the existing world. Therefore, in the conception of an agent knowledge base it must be object of attention the existent information, which may not be known in all its extension; the observed Information, which is determined by the experience, and obtained by contact or observation; and the information to be represented, which refers to a given event and may or may not be taken into consideration.

Definition 1. The knowledge in an agent's knowledge base is made of logic clauses of the form $r_{k}: P_{j+j+1} \leftarrow P_{1} \wedge P_{2} \wedge \ldots \wedge P_{i-1} \wedge$ not $P_{i} \wedge \ldots \wedge$ not $P_{i+j}$, where $i, j$, $k$ belong to the set of natural numbers, $\mathrm{P} 1, \ldots, \mathrm{Pi}+\mathrm{j}$ are literals; i.e., a formula of the form $\mathrm{P}$ or $\neg \mathrm{P}$, where $\mathrm{p}$ is an atom, and where $\mathbf{r}_{\mathbf{k}}$, not, $\mathbf{P}_{i+j+1}$, and $\mathbf{P}_{1} \wedge \mathbf{P}_{2} \wedge \ldots \wedge \mathbf{P}_{\mathbf{i - 1}} \wedge$ not $\mathbf{P}_{\mathbf{i}} \wedge \ldots \wedge$ $\mathbf{P}_{i+j}$ stand, respectively, for the clause's identifier, the negation-by-failure operator, the rule's consequent, and the rule's antecedent. If $\mathrm{i}=\mathrm{j}=0$ the clause is called a fact and is represented as $\mathbf{r}_{\mathbf{k}}: \mathbf{P}_{\mathbf{1}}$. An Extended Logic Program (ELP) program is seen as a set of clauses, as given by the definition below.

Definition 2. An Agent Knowledge Base (AKB) is taken from an ordered theory $\mathrm{OT}=(\mathrm{T},<,(\mathrm{S}, \prec))$, where $\mathrm{T},>, \mathrm{S}$ and $\prec$ stand, respectively, for an $A K B$ in clausal form, a non-circular ordering relation over such clauses, a set of priority rules, and a non-circular ordering relation over such rules.

Definition 3. An argument (i.e., a proof, or series of reasons in support or refutation of a proposition) or arguments have their genesis on mental-states seen as a consequence of the proof processes that go on unceasingly at the agent's own knowledge about its states of awareness, consciousness or erudition.

On the other hand, the mental states are by themselves a product of reasoning processes over incomplete or unknown information; an argument may not only be evaluated in terms of true or false, but it may be quantified over the interval [ $0 \ldots 1]$. An argument may be built over abnormal or exceptional situations and it may recourse to incomplete or contradictory information. This will be accomplished through the use of disjunctive logic programming, here defined in terms of ELP, to the representation of partial information which commonly occurs in the AKBs. We will focus our attention on representing various forms of null values through a set of techniques to distinguish between known and unknown or inapplicable values of attributes in the extensions of the predicates present in the AKBs.

The identification of null values emerges as a strategy for the enumeration of cases, whenever one intends to distinguish between situations where the answers are 
known (true or false) or unknown (unknown) (Traylor and Gelfond, 1993) (Neves et al., 1997). The representation of null values will be scoped by the ELP. In this work, it will be considered two types of null values: the former will cater for the representation of unknown values, not necessarily from a given set of values, and the later will denote unknown values, from a given set of possible values.

Let us consider the predicate reputation(), which stands for an agent reputation and its valuation,

\section{reputation: Entity $\times$ Valuation}

where the first argument denotes the agent and the later its degree of reputation. For example, reputation (Paul, 0.5) denotes that the reputation of the agent Paul has a valuation of 0.5 , a situation that formally may be given in terms of the clauses depicted below (Program 1):

\section{reputation(paul,0.5) \\ -reputation $(E, V) \leftarrow$ not reputation $(E, V)$, \\ exception-to-reputation $(E, V)$}

Program 1 - Extension of the predicate that describes the reputation of agent Paul

In Program 1, the symbol $\neg$ stands for strong negation, not designates negationby-failure, and exception-to-reputation() denotes the set of clauses that are to be considered as exceptions to the extension of predicate reputation(). Considering the example given by Program 1, one may now claim that the reputation of the agent John was not yet been established. This situation will be represented by a null value, of the type unknown, that allows one to conclude that John has a certain reputation, but it is not possible to be concise with respect to its valuation (Program 2).

reputation (paul,0.5)

reputation (john, 1)

$\neg$ reputation $(E, V) \leftarrow$

not reputation $(E, V)$,

not exception-to-reputation $(E, V)$

exception-to-reputation $(E, V) \leftarrow$

reputation $(E, \perp)$

Program 2 - Extension of the predicate that sets the reputation of agent John

The symbol $\perp$ denotes a null value of an undefined type, in the sense that it is assumed that any solution to the problem may be subscribed, but nothing is said about which solution one is speaking about. Computationally, it is not possible to determine, considering the positive information, the reputation of the agent John; however, if one looks to the exceptions to the extension of predicate reputation() (fourth clause of Program 2, that sets the closure of predicate reputation()), it is discarded the possibility of any non-standard question to be assumed as false, when set with respect to the reputation of the agent John.

Consider now the example where the reputation of an agent, the agent Ivan, is foreseen at 0.75 , with a margin of error of $10 \%$. It is not possible to be conclusive regarding the reputation as 0.75 or 0.70 , or even as 0.825 . However, it is false that 
Ivan reputation is 0.70 or 1 . This example suggests that the lack of knowledge may be described by an enumerated set of possible values (Program 3).

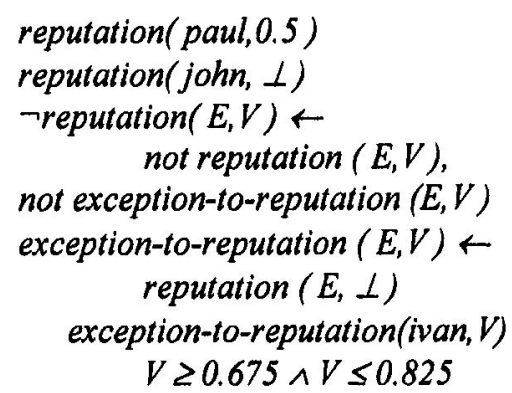

Program 3 - Extension of the predicate that sets the reputation of the agent Ivan

The use of the body of knowledge that makes the AKB, set on the base of the formalisms referred to above, will be pursued in terms of the predicate demo(), using ELP as the logic programming language. Given a question it returns a solution based on a set of assumptions, being defined as:

\section{demo: Question $\times$ Answer}

where Question denotes a theorem to be proved and Answer denotes a truth value, given in the form: true $(t)$, false $(f)$ and unknown $(u)$ (Program 4).

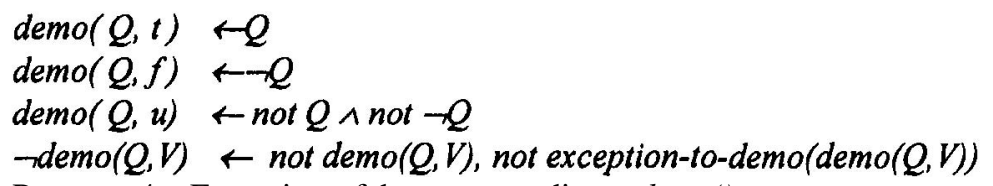

The first clause of Program 4 is used to prove a question using the knowledge base positive information; the second clause is used to reject a question using the knowledge base negative information. The third and fourth clauses stand for themselves.

\section{REPUTATION}

Reputation is the estimation in which a person or thing (e.g., an intelligent agent) is generally held, an opinion with respect to social or professional attitudes such as character, name, good fame, renown, credit or respectability. People may have evil repute or enjoy a good reputation. Reputation can be won or lost, or be obtained from different sources, like the one coming from direct contact or from a third party (Sabater and Sierra, 2002a) (Sabater and Sierra, 2002b). Such esteem is better understood if put into a two dimensional basis, namely:

- An individual dimension, which models the direct interaction between two entities or agents; and

- A social dimension, that may be taken as witness reputation and models the reputation about the target agent coming from its peers; neighbourhood reputation that uses the social environments of the target agents; i.e. uses 
the values of reputation of the neighbourhood with respect to the target agents and their relation with it; and system reputation is the default value of reputation based on the role played by the target agents in the environments.

Reputation may be one important parameter to be taken into account in the process of trust evaluation in a negotiation, once it can be analyzed either in terms of the quality of the information it carries into the negotiation process or the referential it may impose, as a legal issue (Novais et al., 2004).

Based on the assumptions presented before, it is possible to establish mechanisms to analyze and process the information available in a way that turns feasible the study of the behaviour of virtual entities, in terms of its personification. Situations involving forgetfulness, remembrance, learning or trust can be analyzed in the way proposed in this work; i.e., through the description of abnormal situations, declared as exceptions to a predicate extension.

Consider the example that follows, that intends to illustrate, through a case study, the main contributions of this work.

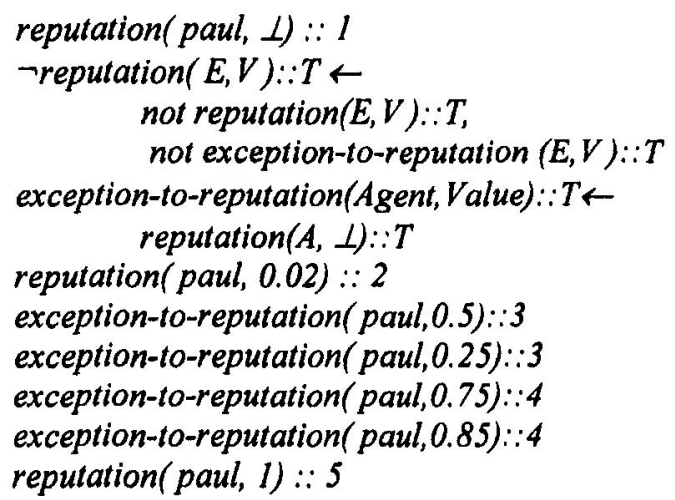

In Program 5, at the point in time 1, there is an axiom stating that the system is aware that Paul has a reputation, but cannot be conclusive (i.e., it is not possible to evaluate the reputation of Paul because there is no information at all to be processed). The second clause of Program 5 enforces that it must be considered false all the questions with lack of information and that cannot be computed in terms of the exceptions to the extension of predicate reputation().

At the point in time 2, there is a proposition that sets the reputation of Paul at 0.02 ; at the point in time 3 , it is known that the reputation of Paul is either 0.5 or 0.25 ; at the point in time 4 , the reputation of Paul is either 0.75 or 0.85 ; at the point in time 5, the reputation of Paul is 1 . Consequently, in terms of the temporal axis $t 1 \rightarrow t 2 \rightarrow t 3 \rightarrow t 4$, an analysis to these values leads one to the conclusion that the $A K B$ learned something (i.e., it acknowledge the Paul's reputation).

In terms of the system semantics, it is intended to evaluate the quality of the information used in a dialog, being it a simple chat or a commercial transaction, by measuring the trustfulness of our peers or opponents. For example, in Program 5, the situation represented by reputation (paul, $\perp):: 1$ corresponds to a case where the quality of the information is given in the form: 


\section{erreputation (paul, $V):: 1=I / N=0$, with $N>>0$}

where $N$ denotes the cardinality of clauses of the form exception-to-reputation( paul,_)::1 (i.e., the clauses that make the exceptions to the extension of predicate reputation ()$)$. The symbol "_" stands for any value that may be endorsed as Paul's reputation. In the situation given by reputation(paul, 0.02) $:: 2$, it corresponds to a case where the quality of the information is given in the form:

$$
\text { erreputation ( paul, } V \text { ) ::2 }=1 / N=0.02 \text {, with } N=50
$$

i.e., the cardinality of the set of the exceptions to the extension of predicate reputation() decreased; it means that the systems is becoming aware of a possible change of course, with respect to the possible moves concerning Paul's reputation.

\section{CONCLUSIONS}

To begin with, it must be said that under most of the western legal systems, computers lack legal personality (i.e., the possibility of being subjects of rights and obligations, of expressing a valid and binding will, of being liable for their own actions). However, intelligent artefacts are not only capable of acting according to its built-in knowledge and rules, but prove to be capable to learn from experience, modify its own states of knowledge, in particular according to cognitive, reactive and pro-active processes quite similar to those of the human beings.

Of course, the consideration of such behaviours and their role - the role of the computer is rapidly evolving from that of passive cipher to that of active participant in the trading process - operates a radical shift in the way we understand basic legal questions such as will and declaration, or the means of manifesting a will in order to get legal effects produced (Portuguese Civil Code, art. $217^{\circ}$ ), which leads us to an imperious need of analysing the question of expression of consent in itself. And two main possibilities have been analyzed: the possibility of considering the electronic devices as mere machines or tools, used by its owner and the daring possibility of considering the electronic device as a legal person.

Therefore, the main contributions of the present work may be understood in terms of the definition of a common ground to situate the agent's reasoning mechanisms in e-The Law environments; the use of formal tools (e.g., Extended Logic Programming) to describe the rational behaviour of the entities involved (e.g., in terms of trust or reputation, declarations of will and agreements); the use of incomplete information and the resource to previous experiences in the reasoning process.

ELP proved to be a well adequate tool for knowledge representation and reasoning, in particular when one intends to endorse situations where the information is vague or incomplete, which is the case when there is the intention to represent at the agent's level properties and attitudes only found in the human beings.

In e-The Law, the introduction of agent-based technology makes possible the use of a set of high-level reasoning tools, leading to architectures that base them on the assumption that in a real-world environment entities act as mediators. Under this umbrella, simple inference and knowledge acquisition mechanisms are not sufficient 
to provide a sustainable and credible system. Traditional computational models need to be complemented with procedures native to the business. Indeed, the issues posed by e-The Law need to be addressed in a formal fashion. Although applications in this area are particularly suited to be solved by agent's based systems, no formal development process has yet been devised for such field of expertise. The processes involved in e-The Law which are difficult to incorporate into traditional systems, revolve around subjective assessments of our parties, opponents, and The Law. Indeed, parameters such as trust and/or reputation among parties are non-linearities, which need to be considered in order to develop a feasible e-The Law system. This information must be taken into account when drawing up a plan of action. However, once subjective parameters have been quantified, some reasoning must take place before any argument is exchanged with potential counterparts. It is at this stage that one deals with the existence of incomplete information and logical conclusions are delineated upon an agent's knowledge base.

\section{REFERENCES}

1. Alferes J, Pereira LM, Przymusinski T. Classical Negation in Nonmonotonic Reasoning and Logic Programming. Journal of Automated Reasoning, vol. 20, pp. 107 142,1998.

2. Allen T, Widdison R. Can Computer Make Contracts?, Harvard Journal of Law Technology, Volume 9, Number 1, Winter 1996.

3. Andrade MD. TeoriaGeral da Relaçãfio Jurídica, Coimbra Editora, vol. I., 1974.

4. Andrade F, Neves J. Intelligent Electronic Inter-systemic contracting-issues on consent and contract formation. Proceedings of the $6^{\text {th }}$ International Conference on Enterprise Information Systems ICEIS 2004, Porto, Portugal, 2004.

5. Arthur W. Inductive Reasoning and Bounded Rationality (The El Farol Bar Problem). Proceedings of the American Economics Review, 84:406,1994.

6. Bazzan A, Bordini R. Evolving agents with moral sentiments in an iterated prisoner's dilemma exercise. Proceedings of the 2nd Workshop on Game Theoretic and Decision Theoretic Agents, 4th ICMAS, 10-12 July, Boston, 13-25,2000.

7. Bazzan A, Bordini R, Vicari R, Wahle J. Evolving populations of agents with personalities in the minority game. Proceedings of the International Joint Conference: 7th IBERAMIA and 15th SBIA, 19-22 November, Atibaia-SP, Brazil, number 1952 in Lecture Notes in Artificial Intelligence, 166175. Berlin: Springer-Verlag, 2000.

8. Castelfranchi C, Rosis F, Falcone R. Social Attitudes and Personalities. In Agents, Socially Intelligent Agents, AAAI Fall Symposium Series 1997, MIT in Cambridge, Massachusetts, November 8-10, 1997.

9. Challet D, Zhang Y. On the Minority Game: Analytical and Numerical Studies, Physica A 256, 514, 1998.

10. Costa M, Neves J. Practical Knowledge Management in the Legal Domain.4th International Conference on Knowledge-based Intelligent Engineering Systems \& Allied Technologies (KES'2000), Sussex, UK, 2000.

11. Costa M, Neves J,Sousa O, Santos S, Verification and Normalization of Sentences, the Seventh International Conference on Artificial Intelligence and Law, June 14-18, Oslo, Norway, 1999.

12. Felliu S. Intelligent Agents and Consumer Protection, in http://www.edip.org.documentsII/ elecagents/consumer_protection.pdf.

13. Fernandes LAC. Teoria Geral do Direito Civil, vol. 1,2nd edition, Lex, Lisboa, 1995.

14. Fischer J. Contracting with electronic agents, in Emory Law Journal, vol. 50, Fall 2001, Nr. 4.

15. Gonçalves C. Tratado de Direito Civil - em comentário ao Código Civil Portugues, Coimbra Editora, 1929, vol. I

16. Lerouge JF. The use of electronic agents questioned under contractual law. Suggested solutions on a European and American level, in http://www.droit.fundp.ac.be/textes/lerouge2.pdf.

17. Miglio F, Odina T, Romano F, Santoro S. Electronic agents and the law of agency in http://www.cirfid.unibo.it/ lea-02/pp/DemiglioOnidaRomanoSantoro.pdf 
18. Neves J. A Logic Interpreter to Handle Time and Negation in Logic Data Bases, in Proceedings of ACM'84, The Fifth Generation Challenge, pp. 50-54,1984.

19. Neves J, Machado J, Analide C, Novais P, Abelha A. Extended Logic Programming Applied to the Specification of Multi-Agent Systems and Their Computing Environments. Proceedings of the 1997 IEEE International Conference on Intelligent Processing Systems, China, 1997.

20. Novais P, Analide C, Machado J, Neves J. Reputation and Trust in the context of Logic-based argumentation. ICKEDS'2004 - International Conference on Knowledge Engineering and Decision Support, Porto, 2004 (to appear).

21. Ortony A, Clore GL., Collins A. The Cognitive Structure of Emotions. Cambridge, UK: Cambridge University Press (1988)

22. Picard R. Affective Computing, MIT Press, Cambridge (1997)

23. Sartor G. Agents in Cyberlaw in http://www.cirfid.unibo.it/ lea-02/pp/Sartor.pdf.

24. Sabater J. and Sierra C., Reputation and social network analysis in multi-agent systems. Proceed-ings of the first International Joint Conference on Autonomous Agents and Multiagent systems, Bologna, Italy, pp. 475-482,2002.

25. Sabater J. and Sierra C., Social ReGreT, a reputa-tion model based on social relations. SIGecom Exchanges. ACM, Vol 3.1, pp 44-56,2002.

26. Traylor B, Gelfond M. Representing Null Values in Logic Programming. Proceedings of the ILPS'93 Workshop on Logic Programming with Incomplete Information, pp. 35 47, Vancouver, Canadá,m 1993.

27. Weitzenboeck E. Electronic Agents and the formation of contracts, in http://www.eclip.org. documentsII/elecagents/contract formation.pdf. 